\section{Controlling enteric nerve cell migration}

A functional gastrointestinal system is dependent on the enteric nervous system, which is formed during embryogenesis through colonization of the gut by enteric neural crest cells (ENCCs). Now, Niswander and colleagues identify the protein phosphatase 1 (PP1)- and actin-binding protein Phactr4 as a regulator of directional and collective ENCC migration (Genes Dev. 26, 69-81; 2012).

Analysis of mouse embryos expressing a Phactr4 mutation known to abolish PP1 binding revealed reduced enteric neuronal numbers and defective organization at embryonic day 18.5, and reduced ENCC numbers in the gut at earlier stages (E12.5). These defects were not due to defective ENCC specification, proliferation or differentiation. Instead, time-lapse imaging of hindgut organ explants revealed disrupted directional migration of ENCCs and detachment of cells from the wave front. Inhibition of PP1 caused similar migration defects in wild-type organ cultures. In wound healing assays, mutant mouse embryonic fibroblasts (MEFs) displayed randomized actin protrusion and migration directions, and reduced lamellipodia size. Phactr4 co-localized with the actin regulator cofilin and integrin- $\beta 1$ at lamellipodia tips, and mutant cells displayed a ROCK-kinasedependent increase in phosphorylation of cofilin and increased integrin- $\beta 1$ signalling.
Interestingly, inhibition of integrin signalling or ROCK activity rescued directed migration of MEFs and normalized the migration of ENCCs in organ cultures. Although the precise function of Phactr4 remains to be discovered, these data demonstrate its role in regulating lamellipodial actin dynamics through cofilin activity controlled by integrin and PP1 signalling. CKR

\section{Actin gets the oxidation treatment from Mical}

Mical, an enzyme mediating redox reactions, is known to promote actin remodelling in response to semaphorin signalling by disassembling actin filaments. However, the precise mechanism of Mical-dependent F-actin disassembly and the identity of Mical substrates have remained obscure. Hung et al. now report that Mical enzymatically modifies a specific actin residue to promote F-actin severing (Science 334, 1710-1713; 2011).

Using in vitro biochemical assays and microscopy techniques, the authors demonstrated that F-actin activates the enzymatic activity of Mical to promote actin filament severing and prevent repolymerization by stably and specifically modifying actin subunits at methionines 44 and 47 through the addition of oxygen. Based on previous knowledge of actin structure, these modifications were predicted to affect the interaction between F-actin subunits.

\title{
Aggregation is key for chimeric monkeys
}

When injected into host mouse blastocysts, mouse pluripotent embryonic stem cells (ESCs) derived from the inner cell mass (ICM) of the embryo, or induced pluripotent cells obtained through reprogramming of somatic cells, can contribute to the formation of all host tissues. Although the preparation of chimera is well-established in mice, it had not yet been achieved in primates. Mitalipov and colleagues demonstrate that aggregation of the totipotent blastomeres of several early dividing Rhesus monkey embryos can successfully yield chimeric monkeys (Cell doi:10.1016/j.cell.2011.12.007; 2012).

In initial experiments, monkey ESCs injected into blastocysts could not incorporate into the host foetuses, indicating a failure of host blastocysts to sustain ESC maintenance. Injected isolated ICMs were also unable to integrate into the host foetuses but could successfully form their own foetus, participate in shared extraembryonic tissue and develop some degree of chimerism with host embryos (mainly in the liver and spleen, perhaps due to the sharing of blood derivatives). Analysis of developmental markers suggested that lineage determination had started in the monkey ICMs, which may have diminished their plasticity and impeded their integration into host foetuses. The authors finally allowed several early dividing and totipotent embryos to aggregate before injection. They obtained foetuses displaying a high degree of chimerism, and healthy chimeric monkeys were born. Interestingly, this set of experiments suggests that it would be difficult to use primate embryos as hosts to test pluripotency by direct injection of candidate pluripotent cells, as cleaving primate embryos does not seem to provide a niche for the maintenance of ESCs.

The authors used point mutations to establish that residue Met 44 of actin was essential for the F-actin-severing function of Mical. Manipulation of Mical levels is known to generate abnormal bristle cell processes in Drosophila. In the present study, mutation of the Met 44 actin residue suppressed Mical overexpression phenotypes and phenocopied Mical loss-of-function effects in Drosophila. Together, these findings establish actin as a direct substrate of Mical and reveal a specific oxidation-dependent mechanism to regulate actin filament dynamics and cell processes in vivo.

AIZ

\section{A mitochondria-lysosome transport pathway}

Reactive oxygen species (ROS) that arise from electron transport chain activity can be neutralized by oxidation of mitochondrial proteins and lipids. However, the way these oxidized macromolecules are removed from the mitochondria is not clear. McBride and colleagues now show that a subpopulation of mitochondria-derived vesicles (MDVs) that are generated in response to oxidative stress are delivered to lysosomes (Curr. Biol. doi:10.1016/j. cub.2011.11.057; 2012). These MDVs might therefore be an important transport intermediate for degrading oxidized mitochondrial proteins.

McBride and colleagues found that MDVs were generated in mammalian cell lines as an early response to oxidative stress. Most of these structures contained the mitochondrial outer membrane protein Tom20, but lacked inner-membrane proteins or matrix-associated proteins. MDV formation did not require the GTPase Drp1 or the autophagy protein ATG5, and the MDVs did not co-localize with autophagosome marker LC3. This indicates that MDV formation is independent of the mitochondrial fission or mitophagy machinery. The authors then traced the movement of these stress-induced MDVs and found that they were delivered to multivesicular bodies, which went on to fuse with lysosomes. Together, these data suggest the existence of a stress-induced mitochondria-lysosome transport pathway that might mediate removal of damaged mitochondrial proteins. $\quad$ EJC

By Emily J. Chenette, Christina Karlsson Rosenthal, Nathalie Le Bot and Alexia-Ileana Zaromytidou 